\title{
Elevated asleep BP as predictor of type 2 diabetes and therapeutic target for prevention
}

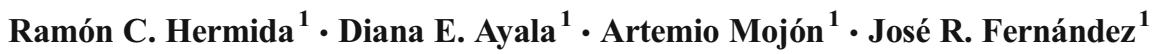

Received: 11 November 2015 / Accepted: 13 November 2015 / Published online: 7 December 2015

(C) Springer-Verlag Berlin Heidelberg 2015

Keywords Ambulatory blood pressure · Chronotherapy · Sleep-time blood pressure · Type 2 diabetes
Abbreviations
ABPM Ambulatory BP monitoring
CKD Chronic kidney disease
CVD Cardiovascular disease
MAPEC Monitorización Ambulatoria para Predicción de Eventos Cardiovasculares
SBP Systolic BP

To the Editor: We read with interest the commentary by Dr Rutter [1] on our two companion papers from the MAPEC (Monitorización Ambulatoria para Predicción de Eventos Cardiovasculares) study, describing, respectively, prognostic value of asleep BP determined by ambulatory BP monitoring (ABPM) [2] and reduced risk of new-onset type 2 diabetes among hypertensive patients ingesting one or more BP-lowering medications at bedtime compared with those ingesting all such medications upon awakening [3]. Beyond the limitations of the study that we openly addressed in our manuscripts, Dr Rutter presents a critical view of our reported findings, to some extent based on speculative assumptions, which requires point-by-point clarification.

Ramón C. Hermida

rhermida@uvigo.es

1 Bioengineering and Chronobiology Laboratories, E.I. Telecomunicación, University of Vigo, Campus Universitario, Vigo 36310, Spain
Point 1 The QDiabetes score (http://www.qdiabetes.org/) is based on patients' and clinical data, including clinic BP, sex, smoking status and BMI, which were also tested as potential confounders in our study. The QDiabetes score, however, does not incorporate fasting plasma glucose concentration or asleep BP mean, which we found to be the two most significant independent prognostic markers of risk for diabetes [2]. Our results document that waist circumference is of significant predictive value for diabetes, whereas BMI (used in QDiabetes only if known) is not. Most important, our findings indicate that clinic BP is of no prognostic value when corrected for asleep systolic BP (SBP) mean. In this regard, our prospective evaluation actually provides novel evidence on the limitations of the QDiabetes score. Dr Rutter also comments on the fact that we did not provide asleep BP thresholds that predict patient risk for new-onset type 2 diabetes in this paper; however, other recent publications of ours do, in fact, recommend differing asleep BP thresholds, not only to diagnose hypertension, but also to assess attainment of clinical goals by pharmacotherapy, for patients both with and without diabetes $[4,5]$.

Point 2 According to current guidelines, 'diabetes may be diagnosed based on $[\mathrm{Hb}] \mathrm{A}_{1 \mathrm{c}}$ criteria or plasma glucose criteria, either the fasting plasma glucose or the 2-h plasma glucose value after a 75-g oral glucose tolerance test' [6]. In cases of discordant test results, diagnosis should rely on the single positive test after confirmation by repeated evaluation. Although our study used only fasting glucose for diagnosis, the diagnosis was consistently validated by repeat measurements at clinical assessments $\geq 3$ months apart [2, 3]. These same guidelines also emphasise the lower sensitivity of $\mathrm{HbA}_{1 \mathrm{c}}$ than fasting glucose for diagnosis, as $\mathrm{HbA}_{1 \mathrm{c}}$ identifies onethird fewer cases of undiagnosed diabetes than elevated fasting glucose [6]. Hence, $\mathrm{HbA}_{1 \mathrm{c}}$ is not a requirement for 
the diagnosis of diabetes, and the fact that it was not used as a diagnostic tool in our studies should not be considered to be a valid limitation.

Point 3 Our first manuscript does not claim causality, but documents an association between elevated asleep SBP mean and increased risk of diabetes [2]. Most important, Dr Rutter in his commentary does not refer to the potentially most relevant finding of the MAPEC trial: that, in addition to the firstdocumented prognostic value of high asleep BP mean, repeated (at least annual) round-the-clock ABPM patient evaluation enabled identification of treatment-induced lowering of asleep SBP mean during follow-up as the most significant marker of reduced risk for future development of diabetes. Evaluation of the effects of treatment-induced changes in ambulatory BP on risk of diabetes by repeated ABPM evaluation means MAPEC is at all levels an interventional, not observational, clinical trial. In addition, there is no scientific proof to Dr Rutter's suggestions that asleep BP elevation might be the result of diabetes; in fact, the novel findings of our trial are just opposite indicating, instead, that elevated asleep BP is a precursor of diabetes. Furthermore, the claim of the commentary [1] that cases of new-onset diabetes in our nearly 6 year median follow-up trial might be patients with undiagnosed diabetes upon recruitment is not only unproven but very surprising, considering the multiple, repeated (at least annual) fasting glucose assessments of every MAPEC participant. In summary, there is no scientific evidence whatsoever on the speculated reverse causality, i.e. that elevated asleep BP is a consequence rather than precursor of diabetes.

Point 4 Our first manuscript [2] clearly states: 'All demographic, anthropometric and clinical laboratory variables listed in Table 1 were tested as potential confounding variables. Adjustments were applied for fasting glucose, waist circumference, age, hypertension treatment-time and chronic kidney disease (CKD) diagnosis, as these influential factors were the only ones consistently significant in all tested Cox regression models.' Thus, despite significant differences between event and non-event patients in most of the variables investigated, including the presence of sleep apnoea and elevated triacylglycerol level at baseline, these variables were not significant independent predictors of outcome. Moreover, physical activity (active/sedentary) was not a significant independent predictor of diabetes in our trial. Finally, our second manuscript [3] states: 'The proportion of patients in the morning and bedtimetherapy groups treated with statins $(16.2 \%$ vs $15.3 \%$, respectively; $p=0.551)$... was also similar.' In summary, we believe there is no evidence that confounding is a limitation of our reported findings, as suggested in the commentary.

Point 5 Although new-onset diabetes was not a pre-specified endpoint in the published trial design $[7,8]$, the MAPEC study was indeed designed to investigate multiple hypotheses [2, 3] associated with multiple risk assessment, including total cardiovascular disease (CVD) morbidity and mortality, major CVD events (composite of CVD death, myocardial infarction, and ischaemic and haemorrhagic stroke) [9, 10], new-onset diabetes, and new-onset and progression of CKD. Randomisation of hypertensive participants to treatment-time (awakening or bedtime) was carried out separately for each allowed hypertension medication, as well as according to sex (male/female), diabetes (present/absent), CKD (present/ absent) and baseline ambulatory BP pattern (dipper/nondipper) to ensure balance of these potentially influential factors between treatment-time groups. In addition, follow-up assessment of all participants was performed at least annually until study closure for the development of new-onset diabetes and/or CKD, independent of corroboration of potentially competitive CVD events. In summary, if diabetes had been the only outcome variable investigated, neither the design nor, in our view, the results of the MAPEC trial would have been any different; thus, we think it unlikely that the findings would have been affected or limited in any way by the evaluation of the specified multiple risks.

Point 6 The commentary states: 'It is easy to imagine that, during the trial, individuals, with knowledge of their differing treatment allocation, could adopt different lifestyle patterns' [1]. Such a speculative assumption, even if it were proved to be true, would only provide evidence of an amazing 'psychological impact' of bedtime hypertension treatment with conventional hypertension medications. If this were the case, this treatment-time regimen, beyond conferring improved efficacy, duration of action, safety and effects on the $24 \mathrm{~h} \mathrm{BP}$ pattern [11], would also need to motivate sufficiently strong compliance to a number of beneficial lifestyle changes, such as weight loss, cessation of alcohol and cigarette smoking, and exercise/fitness programmes. In such a highly hypothetical case, this indeed would only provide further strong evidence that bedtime hypertension treatment must be required as the management of choice for all hypertensive individuals.

Point 7 It is noteworthy that several international medical and scientific societies [5, 12-15] currently acknowledge the clinical relevance of the concept of hypertension chronotherapy by recommending physicians advise patients to ingest $\geq 1$ BPlowering medications at bedtime. Thus, the real issue is not whether this recommendation is a valid one, as questioned in the commentary [1], but rather, whether there is any scientific evidence to support recommending the morning ingestion of hypertension therapy; to our knowledge, no such evidence exists. Beyond the reduction of both CVD [9, 10] and diabetes risks [2] by bedtime hypertension treatment, as found in the MAPEC study, it is of interest that Roush et al [16], who recently compared the results of published studies in which 
hypertension medication was systematically ingested in the evening/bedtime with those in which hypertension medications were ingested daily in the morning, found significant (48\%) better reduction in relative risk of CVD events when hypertension medications are ingested at bedtime rather than in the morning.

In summary, both our papers [2,3] enumerate a number of limitations and strengths of the MAPEC study. We believe the additional assumed limitations described in the accompanying commentary [1] are invalid and do not in any way diminish the scientific merit of our findings. Nonetheless, as previously acknowledged [2,3], future prospective intervention trials that incorporate annual or more frequent around-the-clock ABPM assessments during long-term follow-up evaluation, as carried out in the completed MAPEC study and the currently ongoing multicentre Hygia Project (involving more than 18,000 participants) $[17,18]$, are necessary. This is important, first, to confirm that the asleep SBP mean is a sensitive predictor of risk of diabetes and, second, to determine the merit of bedtime hypertension chronotherapy to avert diabetes through normalisation of asleep SBP mean, which may be mediated by improved effects on those circadian rhythms that regulate in common asleep BP and metabolic processes [19].

Funding The MAPEC study is an independent investigator-promoted research project supported by unrestricted grants from Ministerio de Ciencia e Innovación (SAF2006-6254-FEDER; SAF2009-7028FEDER); Xunta de Galicia (PGIDIT03-PXIB-32201PR; INCITE07PXI-322003ES; INCITE08-E1R-322063ES; INCITE09-E2R322099ES; 09CSA018322PR); and Vicerrectorado de Investigación, University of Vigo.

Duality of interest The authors declare that there is no duality of interest associated with this manuscript.

Contribution statement The authors are jointly responsible for the conception, design, drafting, revision and approval of the letter.

\section{References}

1. Rutter MK (2015) Night-time blood pressure: a role in the prediction and prevention of diabetes? Diabetologia. doi:10.1007/s00125015-3814-2

2. Hermida RC, Ayala DE, Mojón A, Fernández JR (2015) Sleep-time BP: prognostic marker of type 2 diabetes and therapeutic target for prevention. Diabetologia. doi:10.1007/s00125-015-3748-8

3. Hermida RC, Ayala DE, Mojón A, Fernández JR (2015) Bedtime ingestion of hypertension medications reduces the risk of new-onset type 2 diabetes: a randomised controlled trial. Diabetologia. doi: 10 . 1007/s00125-015-3749-7

4. Hermida RC, Ayala DE, Mojón A, Fernández JR (2013) Ambulatory blood pressure thresholds for diagnosis of hypertension in patients with and without type 2 diabetes based on cardiovascular outcomes. Chronobiol Int 30:132-144
5. Hermida RC, Smolensky MH, Ayala DE et al (2013) 2013 Ambulatory blood pressure monitoring recommendations for the diagnosis of adult hypertension, assessment of cardiovascular and other hypertension-associated risk, and attainment of therapeutic goals. Joint recommendations from the International Society for Chronobiology (ISC), American Association of Medical Chronobiology and Chronotherapeutics (AAMCC), Spanish Society of Applied Chronobiology, Chronotherapy, and Vascular Risk (SECAC), Spanish Society of Atherosclerosis (SEA), and Romanian Society of Internal Medicine (RSIM). Chronobiol Int 30:355-410

6. American Diabetes Association (2015) Classification and diagnosis of diabetes. Diabetes Care 38(Suppl 1):S8-S16

7. Hermida RC, for the MAPEC Study Investigators (2007) Ambulatory blood pressure monitoring in the prediction of cardiovascular events and effects of chronotherapy: rational and design of the MAPEC Study. Chronobiol Int 24:749-775

8. Hermida RC, Ayala DE, Mojón A, Fernández JR (2015) Erratum to: bedtime ingestion of hypertension medications reduces the risk of new-onset type 2 diabetes: a randomised controlled trial. Diabetologia. doi:10.1007/s00125-015-3806-2

9. Hermida RC, Ayala DE, Mojón A, Fernández JR (2011) Decreasing sleep-time blood pressure determined by ambulatory monitoring reduces cardiovascular risk. J Am Coll Cardiol 58:1165-1173

10. Hermida RC, Ayala DE, Fernández JR, Mojón A (2013) Sleep-time blood pressure: prognostic value and relevance as a therapeutic target for cardiovascular risk reduction. Chronobiol Int 30:68-86

11. Hermida RC, Ayala DE, Fernández JR et al (2013) Administrationtime-differences in effects of hypertension medications on ambulatory blood pressure regulation. Chronobiol Int 30:280-314

12. American Diabetes Association (2012) Standards of medical care in diabetes-2012. Diabetes Care 35(Suppl 1):S11-S63

13. The Task Force of diabetes, pre-diabetes, and cardiovascular diseases of the European Society of Cardiology (ESC) and European Association for the Study of Diabetes (EASD), Rydén L, Grant PJ et al (2013) ESC guidelines on diabetes, pre-diabetes, and cardiovascular diseases developed in collaboration with the EASD. Eur Heart J 34:3035-3087

14. Shimamoto K, Ando K, Fujita T et al (2014) The Japanese society of hypertension guidelines for the management of hypertension (JSH 2014). Hypertens Res 37:253-390

15. Chiang CE, Wang TD, Ueng KC et al (2015) 2015 guidelines of the Taiwan society of cardiology and the Taiwan hypertension society for the management of hypertension. J Chin Med Assoc 78:1-47

16. Roush GC, Fapohunda J, Kostis JB (2014) Evening dosing of antihypertensive therapy to reduce cardiovascular events: a third type of evidence based on a systematic review and meta-analysis of randomized trials. J Clin Hypertens (Greenwich) 16:561-568

17. Ayala DE, Moyá A, Crespo JJ et al (2013) Circadian pattern of ambulatory blood pressure in hypertensive patients with and without type 2 diabetes. Chronobiol Int 30:99-115

18. Moyá A, Crespo JJ, Ayala DE et al (2013) Effects of time-of-day of hypertension treatment on ambulatory blood pressure and clinical characteristics of patients with type 2 diabetes. Chronobiol Int 30:116-131

19. Fabbian F, Smolensky MH, Tiseo R, Pala M, Manfredini R, Portaluppi F (2013) Dipper and non-dipper blood pressure 24-hour patterns: circadian rhythm-dependent physiologic and pathophysiologic mechanisms. Chronobiol Int 30:17-30 